\title{
A Novel Optimization of the Distance Source Routing (DSR) Protocol for the Mobile Ad Hoc Networks (MANET)
}

\author{
Syed S. Rizvi ${ }^{1}$, Majid A. Jafri, and Khaled Elleithy \\ Computer Science and Engineering Department \\ University of Bridgeport \\ Bridgeport, CT 06601 \\ \{srizvi,majidals,elleithy\}@bridgeport.edu
}

\author{
Aasia Riasat \\ Department of Computer Science \\ Institute of Business Management \\ Karachi, Pakistan 78100 \\ aasia.riasat@iobm.edu.pk
}

\begin{abstract}
This paper presents a new scheme for the Distance Source Routing (DSR) protocol which shows the improvement over the two major metrics of the DSR protocol: Route Discovery and Route Maintenance. In addition, we present a mathematical model that includes probability density function for these two observed metrics. Our simulation results demonstrate a significant improvement in the route discovery, transmission time, and the overall network utilization. As an interesting side result, our analysis also shows that the proposed model can be used to effectively reduce the packet losses.
\end{abstract}

Keywords- DS-CDMA, bit error rate, data throughput, multiuser communications

\section{INTRODUCTION}

The Dynamic Source Routing (DSR) protocol is dealt under On-Demand Routing (ODR) protocol which is just an exact opposite to the Table-Driven Routing (TDR) [2, 3]. Generally, there are two main phases use in the DSR protocol. One is the Route Discovery (RD) phase which discovers all the possible paths for the packets to be transferred from a particular source to a destination. It is essential to properly maintain the $\mathrm{RD}$ phase since maintaining a separate table for storing routing details involves cost issues. The second phase of the DSP protocol is the Route Maintenance (RM) phase which fixes all the possible paths from one particular source to a destination [5]. In DSR, the packets are transmitted only one time for each node. If the node does not receive the packet, the previous node is responsible to make attempts in order to transmit the packet. On the other hand, if the destination node receives the packet successfully, an acknowledgment is transmitted back to the source node for the received packet. Since the use of the DSR protocol does not require the maintenance of a cache table, it allows us to avoid unnecessary updating works which results space and time saving advantages.

In the existing DSR scheme, the malfunctioning of one or more links along a certain route requires the retransmission of all packets back to the originating source node. This unnecessary amount of retransmission results a significant transmission overhead that can severely degrade the overall network performance by increasing the average time delay. In order to minimize the transmission overhead and maximize the network throughput, we present an alternative scheme that can be used to optimize the performance of DSR protocol. Specifically, our proposed scheme suggests improvement in the RD and the RM metrics of the DSR protocol. Based on the proposed optimization, we derive a mathematical model which proves the correctness of the proposed scheme.

\section{Proposed Optimization For The DSR Protocol}

Our main goal is to maintain the original underlying architecture of the DSR protocol. Therefore, we consider the DSR scheme as a black box. The DSR protocol fails to maintain route consistency in the presence of broken links. When one of the links goes down, the DSR protocol locates an alternate route and transmits back the packet to the source node where the packet was originated. On contrary to the actual scheme of the DSR protocol, our proposed scheme uses a reserve direction search method. In our proposed scheme, the packets would be transmitted to the immediate prior node where the actual error was occurred. The proposed scheme then finds one or more alternative routes from the current location to the destination. This implies that the whole searching procedure of the proposed scheme will

${ }^{1}$ Contact author: srizvi@ bridgeport.edu, 


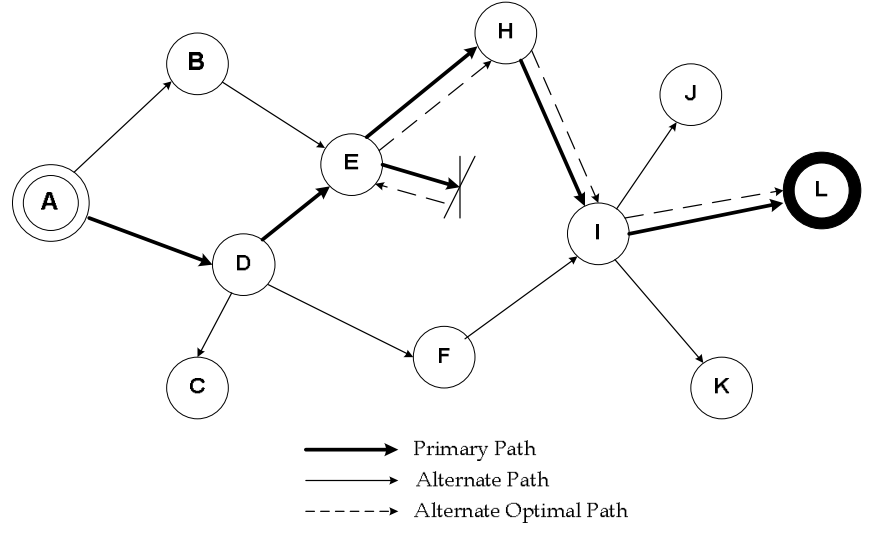

Fig.1. Finding the alternate path in DSR protocol according to the proposed scheme

be done in the opposite direction starting from the destination node. Our simulation results demonstrate that the proposed scheme considerably increases the chance of finding a valid route for salvage packets that are typically stored in the send buffer.

For instance, consider an example for locating a route based on the reverse direction search scheme as shown in Fig. 1. It can be observed that the route finds by the RD procedure from node $A$ (source node) to $L$ would be: $\mathrm{A} \rightarrow \mathrm{D} \rightarrow \mathrm{E} \rightarrow \mathrm{I} \rightarrow \mathrm{L}$. During transmission of the packets, it is detected at run time that the shortest link between node $E$ and $I$ goes down. Consequently, the proposed scheme immediately starts searching the best available alternate routes. In order to reach the destination node, the proposed scheme locates the neighboring nodes (i.e., node $B, D$, and $H$ from node $E$ ). This process of finding the alternate route from the location of error results an optimal alternate route: $A \rightarrow D \rightarrow E \rightarrow I \rightarrow H \rightarrow L$. This implies that our proposed scheme neither send any feedback to the destination node $A$ nor it initiates the route discovery from the source point. Therefore, repeating this search in the reverse direction from the current location of error to the neighboring nodes results a significant increase in the chance of finding a valid optimized route.

\section{A. Proposed Reverse Direction Search Scheme}

In order to formulate the proposed scheme, we present a model that shows simple steps that need to be implemented for finding a valid and optimize route in the presence of link failures. The model is presented in Fig. 2. The model is typically divided into two parts. The upper part of the model represents the $\mathrm{RD}$ procedure where as the lower part represents the RM procedure. The RD procedure is based on an exhaustive search of an internal cache. During the transmission of a packet, if one of the links goes down, the proposed scheme mentions that the packet will be

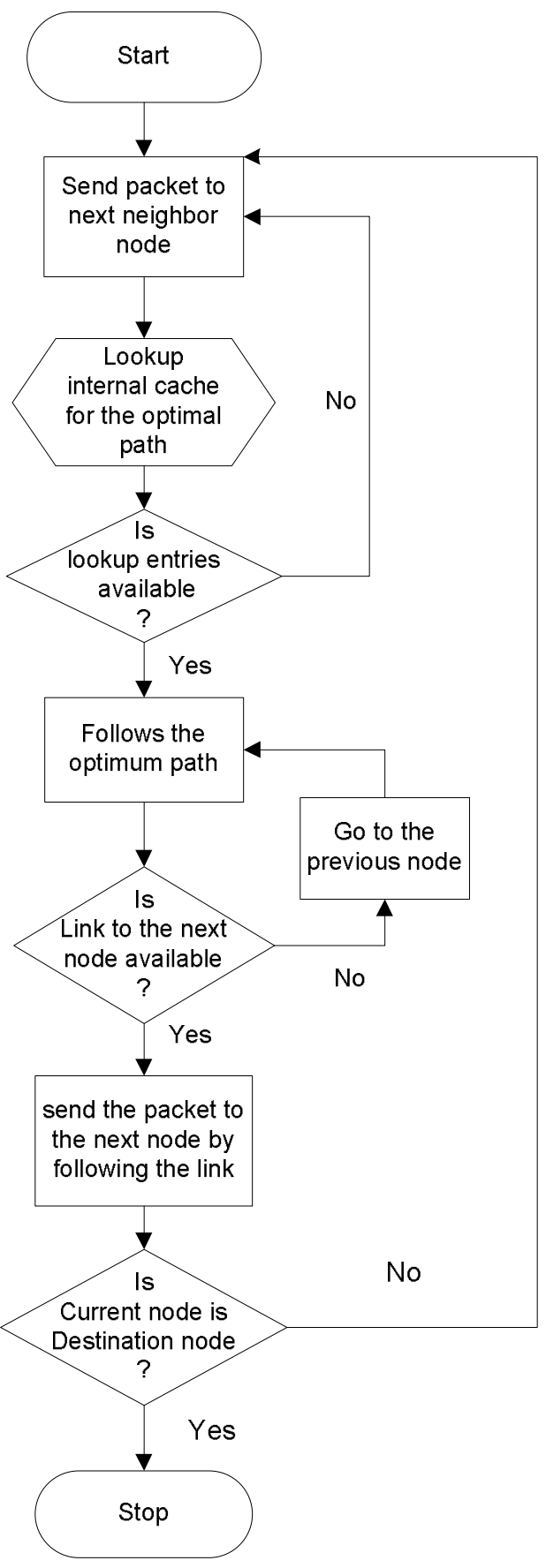

Fig. 2. Flow chart showing proposed model of DSR algorithm

immediately forwarded to the next available node and starts transmitting from the new location. Unlike the DSR protocol, the proposed scheme minimizes the transmission overhead by avoiding the unnecessary transmission of data to the source node in the presence of a faulty link. In other words, the proposed scheme does not provide any feedback to the source node that leads to a significant improvement in the network throughput. Since the RD can be done on the current node, we do not need to focus on the source node. This implies that the proposed scheme suggests the best 
delivery of the packets even in the presence of link failure. In addition, the repetition of the packets due to the flooding will be cut down.

In the proposed model, we mainly focus on the RD and the RM. During the RD process, if the entries are found in the internal cache of the next node, the proposed scheme determines the optimal path that will be used to forward all the packets to the next node. At that current node location, the same procedure for searching the optimal path will be repeated over the passage of time in order to find the best path towards the destination. An empty entry in the internal cache represents that there is no valid route exist for a particular destination. In such a scenario, the proposed scheme will lookup into the next neighbor's cache and determine the best available route for the desired destination. Once the optimal route is discovered, the packet can then be transmitted. In the RM process, whenever there is a link failure along the path, the packet would not go further at the point of error and there is no need to send any feedback to the original source node. Instead, the proposed scheme determines and performs the RM process on the best available alternate path.

\section{B. Mathematical Model}

We derive our mathematical model based on the proposed reverse direction scheme. In our mathematical model, we show that the transmission of packets via an alternate route is more efficient as compared to transmitting packets from the source node using a primary route. This is especially true in the presence of error. All system variables, along with their definition, are listed in Table I.

The accuracy of the proposed scheme is essentially dependent on how efficiently we can discover the alternate routes in the presence of faulty links. In general, the accuracy is partially related to a certain interval by which we perform the RD procedure for a specific type of network traffic such as a stream of packets. In particular, we first need to derive an expression for a random variable, $x$, that can be used to characterize the behavior of RD process with respect to time. Therefore, in order to implement the proposed scheme, one must measure the frequency of route discoveries. In order to determine the interval between the route discoveries, the following mathematical expression can be derived for a random variable, $x$ :

$$
\int_{-\infty}^{+\infty} x f(x) d x
$$

It should be noted that equation (1) is based on the PDF which is used to find the frequency of route discovery for a particular pair of source and destination.

Figure 4 represents the proposed scheme with the primary and the secondary paths along with their corresponding links. It can be seen in Fig. 3 that the node $P$ represents the primary route whereas the node $S$ represents the secondary
TABLE I

\begin{tabular}{|l|l|}
\hline \multicolumn{1}{|l}{ SYSTEM PARAMETERS AND DEFINITIONS } \\
\hline$P_{i}$ & Description \\
\hline$S_{i}$ & This represents the ith link in a primary path. \\
\hline$X_{P i}$ & This represents the ith link in a secondary path. \\
\hline$X_{S i}$ & Life time of the ith primary route. \\
\hline$X_{R}$ & Life time of the ith secondary route. \\
\hline$T$ & $\begin{array}{l}\text { Minimum life time for the collection of all values in } \\
\text { the primary path links }\end{array}$ \\
\hline$E_{o}$ & Intervals for route discovery \\
\hline$f_{T}(t)$ & An event that shows any of the given link fails \\
\hline$Z_{i}$ & Frequency of route discovery \\
\hline $\bar{P}_{i}$ & Maximum life time among all available values. \\
\hline $\bar{S}_{i}$ & Represents the faulty primary link due to an event $E$ \\
\hline
\end{tabular}

route. If an error occurs in the primary route, the proposed scheme will immediately discover an alternate route $S_{1}$ rather than going back to the source node $A$. In other words, in the presence of faulty links, the proposed scheme searches the internal cache and determines the alternative route $S_{1}$ which is typically stored in the local cache.

For this particular scenario, the success of the proposed scheme is heavily dependent on the rate at which one may need to execute the RD procedure. In addition, the success of the proposed scheme is not only dependent on the rate at which the RD procedure will be performed but also dependent on the accuracy and the efficiency by which the alternate routes will be determined. In order to find the frequency of an alternative RD, we assume that an event $E$ might occur at a discrete point in time in the network which causes an error in one of the two types of routes (i.e., the primary $P$ and the secondary $S$ routes). Thus the transmission of an event can be mathematically described as:

$$
E=\overline{P_{1}} \overline{S_{1}}+\left(\overline{P_{2}}\left(\bar{P}_{1}+\overline{S_{2}}\right) \overline{S_{1}}\right)+\left(\overline{P_{3}}\left(\bar{P}_{1}\left(\overline{P_{2}}+\overline{S_{3}}\right) \overline{S_{2}}\right) \overline{S_{1}}+\ldots\right.
$$

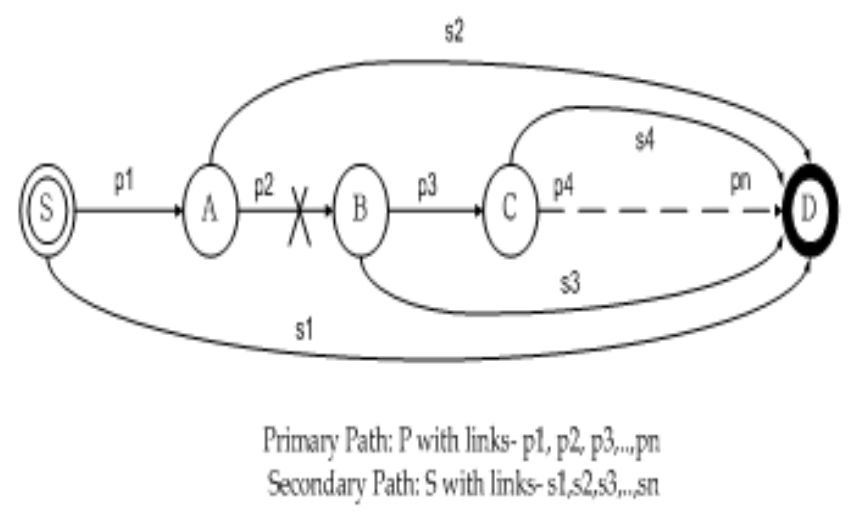

Fig. 3: Proposed scheme with primary and secondary path and their links 
where $\bar{P}_{i}$ represents the faulty primary-link where as $\overline{S_{i}}$ represents the faulty secondary-link which caused due to the occurrence of an event $E$ at discrete point in time within a network.

Equation (2) represents a generic equation that shows how the occurrence of an event in the network may cause an error in the alternate routes. Equation (2) can be further extended for the maximum $K$ number of forwarding links within the available primary paths. It should be noted that the occurrence of an event $E$ is representing a cause of malfunctioning in the currently used valid route. Taking these factors into account, one may write the following mathematical expression:

$$
\begin{aligned}
& E=\left\langle\overline{P_{1}} \overline{S_{1}}\right\rangle+\left\langle\overline{P_{2}} \overline{S_{2}} \overline{S_{1}}\right\rangle+\left\langle\overline{P_{3}} \overline{S_{3}} \overline{S_{2}} \overline{S_{1}}\right\rangle \\
& +\ldots+\left\langle\overline{P_{k}} \overline{S_{k}} \overline{S_{k-1}} \overline{S_{1}}\right\rangle
\end{aligned}
$$

where $\bar{P}_{i}$ and $\overline{S_{i}}$ in (3) represent the faulty primary and secondary links, respectively. Both of these faulty links are caused due to the occurrence of an event $E$ at a discrete point in time within a network. It should be noted that we only consider the values of the most forwarding links that one may find within the primary path links from the generic equation (2).

One of our observations about the two phases of the proposed scheme is the life time of the primary path which we use to transmit the packets to the desired destination in the presence of the faulty links. In other words, in order to effectively implement the proposed scheme, we must determine the minimum value of the life time for primary path links. This calculation is essential, since the ration of determining the accurate valid primary links is critically dependent on the knowledge of accurate values of lifetime. The minimum life time of primary path links is simply chosen from one of the primary links that has a smallest value for the life time. In other words, if one of the ith primary routes has the smallest life time value, this will be chosen as a minimum life time value for the primary path links. This hypothesis can be changed into a simple expression:

$$
X_{R}=\operatorname{Min}\left|X_{p 1}, X_{p 2}, \ldots ., X_{p k}\right|
$$

where $X_{R}$ in (4) represents the minimum life time value for the collection of all values in the primary path links. The right hand side expression of (4) represents the life time of each individual primary route starting from $X_{p l}$ to $X_{p k}$. These values are considered as a life time of the sub links in the primary path. Similar to (4), we can further extend our mathematical model for computing the interval of time for the $\mathrm{RD}$ procedure:

$$
T=\operatorname{Max}\left|X_{s 1}, X_{s 2}, \ldots, X_{s n}\right|
$$

where $T$ represents the intervals of time for the RD and $X_{S i}$ represents the life time of the $i t h$ secondary route.

Equation (5) gives an estimate of the time to be taken by the proposed scheme for the RD procedure. This value is evaluated from the maximum values of the collected time in the sub links of the secondary path. The right hand side expression of (5) represents the life time of each individual secondary route starting from $X_{s I}$ to $X_{s n}$. For the sake of the simulation and the performance evaluation, we assume that the value of $T$ will be measured in millisecond. Combining (4) with (5), we can compute the value of the alternative route discovery as follows:

$$
T=\operatorname{Min}\left\langle\begin{array}{l}
\operatorname{Max}\left(X_{p 1} \cdot X_{s 1}\right), \operatorname{Max}\left(X_{p 2} . X_{s 2} . X_{s 1}\right) \\
\ldots \operatorname{Max}\left(X_{p k}, X_{s k}, X_{s k-1}, \ldots . X_{s 1}\right)
\end{array}\right\rangle
$$

Equation (6) gives the value of the alternative RD. This can be considered as the optimum value which is determined from all the available maximum values for both the primary and the secondary links. Using (6), we can compute the values for the RD metrics which is one of the subparts of the proposed scheme.

$$
Z_{i}=\operatorname{Max}\left\langle X_{p i}, X_{s}, X_{s i-1}, \ldots, X_{s 1}\right\rangle
$$

where $Z_{i}$ represents the maximum life time among all available values for both primary and the secondary paths.

Recall (1), we can now derive an expression for the frequency of RD using equations (2) to (6).

$$
f_{T}(t)=\sum_{i=1}^{N}\left(\lambda_{i} e^{-\lambda_{i} t} \prod_{k=1}^{N}\left(1-e^{-\lambda_{k} t}\right)\right)
$$

where the right hand side of (8) represents the frequency of $\mathrm{RD}$.

Equation (7) also has a significant impact on the RD for the alternate path. Implementing the results of (7) on (8), we can derive a new expression for the frequency of the RD which take into account the maximum life time among all available values for both primary and the secondary paths. In addition, this implementation describes the PDF in $Z_{i}$ with respect to the RD metrics. 


$$
f_{Z i}(t)=\sum_{j=1}^{i+1}\left\langle\begin{array}{c}
\left(\lambda_{j}(i) e^{-\lambda} j^{(i)} t\right) \\
\prod_{k=1, k \neq j}^{i+1}\left(1-e^{-\lambda} k^{(i)} t\right)
\end{array}\right\rangle
$$

where $\lambda_{j}^{(i)}=k i / l \rightarrow$ for $j=1,2 \ldots . i$ and for $1 / l \rightarrow$ $j=i+1$.

Equation (9) describes the summation of all the possible routes which can lead us to the desired destination. Equation (9) can be further extended for the following given expressions:

$T=\operatorname{Min}\left\langle\begin{array}{l}\operatorname{Max}\left(X_{p 1} . X_{s 1}\right), \operatorname{Max}\left(X_{p 2} . X_{s 2} . X_{s 1}\right) \\ \ldots \operatorname{Max}\left(X_{p k}, X_{s k}, X_{s k-1}, \ldots . X_{s 1}\right)\end{array}\right\rangle$

$Z_{i}=\operatorname{Max}\left(X_{p i}, X_{s i}, X_{s i-1} \ldots X_{s 1}\right)$

$T=\operatorname{Min}\left(Z_{1}, Z_{2}, Z_{3} \ldots Z_{k}\right)$

Based on the above three expressions, we can approximate the PDF of $T$ for the frequency of RD as follows:

$$
f_{T}(t)=\lim _{d t \rightarrow 0} p[t \leq T \leq t+d t] / d t
$$

Equation (10) gives the value for the frequency of the RD in terms of a PDF function. Relating (8) and (9) with (10), we can derive the following mathematical expression

$$
\begin{aligned}
f_{T}(t) & =\sum_{i=1}^{k} f_{Z i}(t) \prod_{j=1, j \neq 1}^{k} p\left[z_{j}>z_{i}\right] \\
f_{T}(t) & =\sum_{i=1}^{k} f_{Z i}(t) \prod_{j=1, j \neq 1}^{k}\left(1-F z_{i}(t)\right)
\end{aligned}
$$

where, $F_{z i(t)}$ in (11) was introduced from (7) to make $Z_{i}$ as a function of PDF.

Equation (11) shows that we derived the expected expression which can be used to compute the interval between the rout discoveries. In other words, one could use (11) to determine the frequency of the alternate RD process. The same frequency value can be used to measure the efficiency of the network. In addition, the final results show that the use of the proposed reverse direction scheme with the derived mathematical model can effectively minimize the transmission delay especially in the presence of collisions (links error) or faulty links due to the malfunctioning.

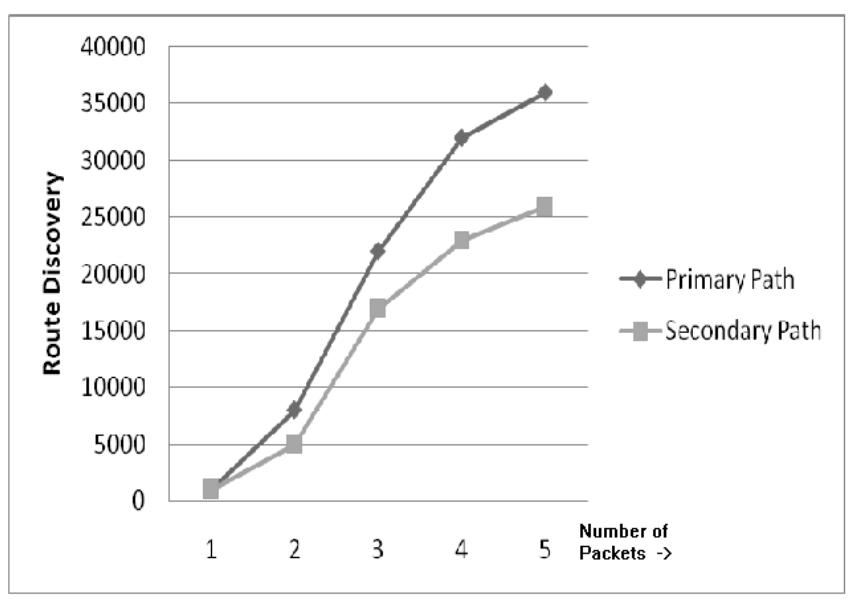

Fig.4. Number of nodes versus RD

\section{SimUlation ReSUlTS}

We simulate our model based on the predicted data from the existing DSR model suggested in [1, 4]. For the sake of simulation and the performance evaluation, we consider two major metrics for RD and RM. These metrics are considered for the evaluation of the efficiency of a network.

For the sake of the first simulation (see Fig. 4), we characterize the behavior of the RD phase of the proposed scheme with respect to the number of nodes present in the network. The purpose of this experiment is to show the performance of the RD phase for discovering the alternate primary and the secondary path. During the simulation, we consider that as the number of nodes increases in the network, the more packets will be accumulated in the network that could affect the performance of the RD phase. It can be clearly evident in Fig. 4 that the RD phase of the proposed scheme performs better for the primary paths discoveries than for the secondary path. When we have small

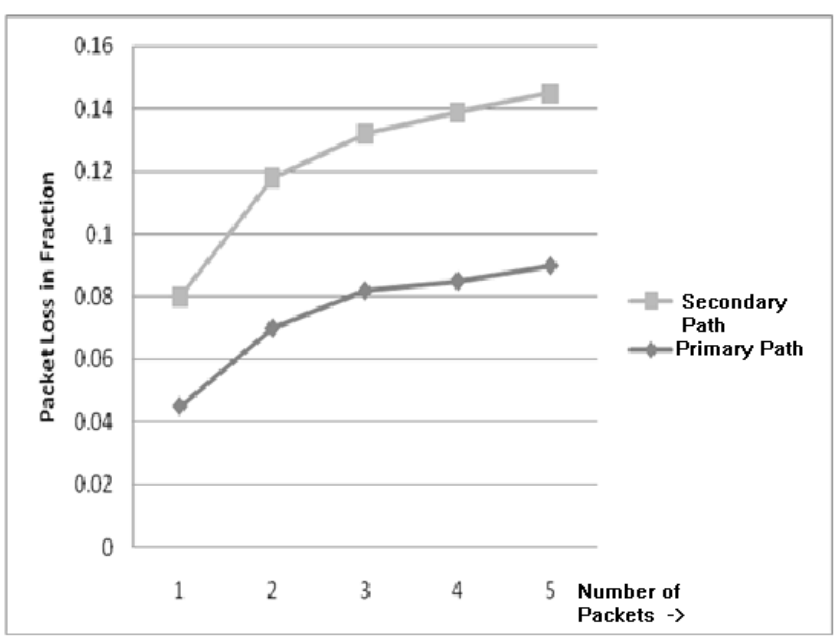

Fig. 5. Packet loss in fractions versus number of nodes 


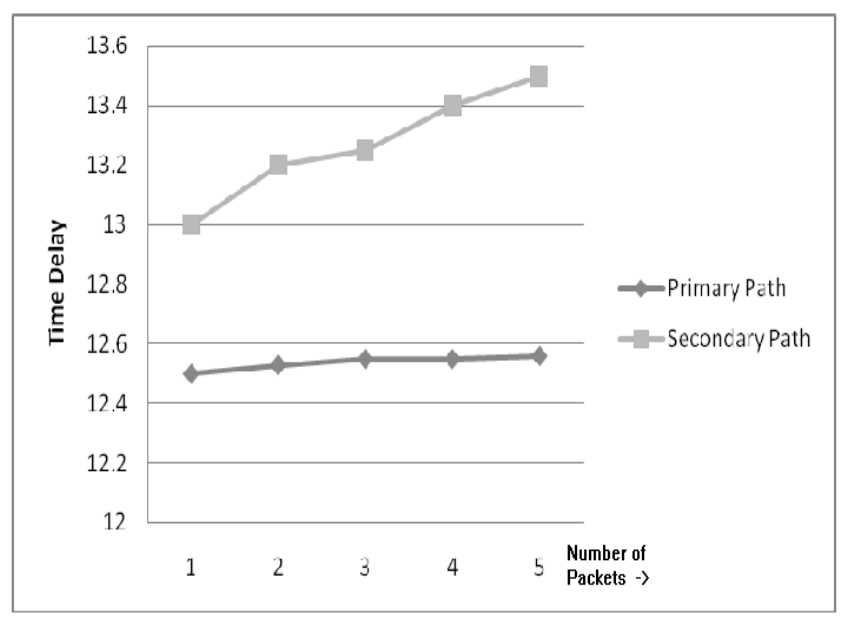

Fig. 6. Time delay versus number of nodes

number of nodes, it can be seen in Fig. 4 that the performance of the RD phase for both primary and secondary path discoveries is overlapping. However, as network grows in terms of the number of nodes, the performance differences between the primary and the secondary path is obvious.

Fig. 5 shows the packet losses (in the fraction value) with respect to the number of nodes during the transmission using both primary and the secondary paths. In addition, Fig. 6 represents a comparison between the time delay (represents in seconds) and the number of nodes. It can be seen in Fig. 6 that the time required to discover the primary paths using the $\mathrm{RD}$ phase is very low as compared to the time required to discover the secondary paths.

Based on the simulation results of Fig. 6, we can observe that the time delay for primary paths is not only small but also linear with respect to the number of nodes. In other words, when we increase the number of nodes in the network, more packets will be accumulated that make a linear increase in the time delay for discovering the secondary paths which is not really desirable as far as the optimum performance of the DSR protocol is concerned.

\section{CONCLUSION}

In this paper, we presented a new scheme that improves the retransmission mechanism for the existing DSR protocol. In order to support our hypothesis, we provided a complete mathematical model that shows the formulation of the proposed scheme. In particular, we investigated the RD and the RM phases with respect to the proposed reverse direction scheme. We also showed that how effective the proposed scheme would be when we implement it with the reverse direction search for discovering the primary paths. Our analysis also suggested that the discovery of alternate primary paths from the current source of error significantly improves the network performance in terms of RD process, time delay, and the packet losses. Moreover, we have experimentally verified that both the RD and the RM metrics perform well with the proposed scheme than the existing infrastructure of the DSR protocol. Our performance evaluation is also well supported by the simulation results presented in this paper.

\section{REFERENCES}

[1] P. Papadimitratos and Z. Haas, "Secure Routing for Mobile Ad hoc Networks," In Proceedings of the SCS Communication Networks and Distributed Systems Modeling and Simulation Conference (CNDS 2002), San Antonio, TX, January 27-31, 2002.

[2] J. Raju and J. Garcia-Luna-Aceves, "A comparison of on demand and table driven routing for ad-hoc wireless networks," In Proc. IEEE International Conference on Communications (ICC 2000), June 2000. Volume 3, Issue 2000, pp. 1702 - 1706, 2000.

[3] J. Raju and J. Garcia-Luna-Aceves, "Efficient On-Demand Routing Using Source-Tracing in Wireless Networks," In Proc IEEE Global Telecommunications (GLOBECOM 2000), Vol. 1, Issue 2000, pp. 577 581, November 2000.

[4] B. Johnson, A. Maltz, and Y. Chun, "The Dynamic Source Routing Protocol for Mobile Ad Hoc Networks. (DSR)," IETF INTERNET DRAFT, 24 February 2003.

[5] V. Park and M. Corson, "A Highly Adaptive Distributed Routing Algorithm for Mobile Wireless Networks," Sixteenth Annual Joint Conference of the IEEE Computer and Communications Societies. Driving the Information Revolution (INFOCOM '97), pp.1405, 1997. 